\title{
Chronic Liver Disease
}

National Cancer Institute

\section{Source}

National Cancer Institute. Chronic Liver Disease. NCI Thesaurus. Code C113609.

Hepatic necrosis, inflammation, or scarring due to any cause that persists for more than

6 months. Manifestations may include signs and symptoms of cholestasis, portal hypertension, and/or abnormal liver function tests. 